\title{
Les femmes juives dans le premier mouvement féministe allemand (1865-1933)
}

La question de l'identité et de la différence au féminin

Jüdische Frauen in der ersten deutschen Frauenbewegung (1865-1933) weibliche Identität und Differenz

Jewish Women in the First German Women's Movement (1865-1933): The Question of Identity vs. Difference

Anne-Laure Briatte

\section{(2) OpenEdition}

\section{Journals}

Édition électronique

URL : https://journals.openedition.org/ceg/7656

DOI : 10.4000/ceg.7656

ISSN : 2605-8359

Éditeur

Presses Universitaires de Provence

\section{Édition imprimée}

Date de publication : 25 septembre 2019

Pagination : 185-197

ISBN : 979-10-320-0234-6

ISSN : 0751-4239

Référence électronique

Anne-Laure Briatte, «Les femmes juives dans le premier mouvement féministe allemand

(1865-1933) », Cahiers d'Études Germaniques [En ligne], 77 | 2019, mis en ligne le 25 mars 2021, consulté le 15 juin 2021. URL : http://journals.openedition.org/ceg/7656 ; DOI : https://doi.org/ $10.4000 /$ ceg.7656 


\title{
Les femmes juives dans le premier mouvement féministe allemand (1865-1933)
}

\author{
La question de l'identité et de la différence au féminin
}

\author{
Anne-Laure BRIATTE \\ Sorbonne Université / SIRICE (UMR 8138)
}

En gestation depuis la révolution de 1848, le premier mouvement féministe allemand est entré dans une phase de constitution en 1865 avec la création à Leipzig de l'Allgemeiner Deutscher Frauenverein (ADF - Association générale des femmes allemandes) par Louise Otto-Peters (1819-1895) et Auguste Schmidt (1833-1902). Au-delà de la diversité des objectifs des associations féminines qui le constituaient, il luttait pour que l'appartenance au sexe féminin ne soit plus génératrice de différences au détriment des femmes, aussi bien dans l'accès au savoir et au marché du travail, que dans le droit conjugal et familial, dans les droits civiques, parmi lesquels le droit de vote, celui d'élire et d'être élue ${ }^{1}$. Ce mouvement était constitué de femmes très majoritairement issues des milieux bourgeois cultivés d'orientation libérale. Les travailleuses n'en faisaient pas partie ; pour cette raison, lorsqu'il sera question du mouvement féministe dans ce qui suit, c'est au seul mouvement féministe « bourgeois » qu'il sera fait référence.

Le premier mouvement féministe allemand comptait parmi ses membres un nombre considérable de femmes juives. Les termes "femme » et " juive » renvoient respectivement à l'appartenance à un sexe (sex en anglais) et l'appartenance religieuse, deux catégories génératrices de différences - à côté de celles de l'appartenance sociale (class), de l'ethnie (race) et d'autres encore, selon les théories de l'intersectionnalité ${ }^{2}$. Leconcept d'« intersectionnalité », utilisé la première fois en 1989 et théorisé depuis par la juriste afro-américaine Kimberlé Crenshaw, a induit un renouvellement des études sur le genre dans les années

1. On parle de premier mouvement féministe allemand de 1865 à 1933, date à laquelle le pouvoir national-socialiste a mis fin au projet émancipateur des femmes. Patrick Farges, Anne-Marie Saint-Gille (dir.), Le premier féminisme allemand 1848-1933 : un mouvement social de dimension internationale, Villeneuve d'Ascq, PU du Septentrion, 2013 ; Angelika Schaser, Frauenbewegung in Deutschland 1848-1933, Darmstadt, Wissenschaftliche Buchgesellschaft, 2006.

2. Fait intéressant, la théorie de l'intersectionnalité avant la lettre est née lors d'un congrès féministe nord-américain en 1851, lorsque l'esclave émancipée Sojourner Truth (env. 1797-1883), face aux résistances des féministes blanches à lui donner accès au pupitre parce qu'elle était noire, a posé la question au public : « Ain't I a woman? ». Katrin Meyer, Theorien der Intersektionalität zur Einführung, Hamburg, Junius, 2017, p. 9. 
1990 et est discuté depuis une dizaine d'années dans les sciences historiques, où des historien.ne.s plaident pour une approche intersectionnelle ${ }^{3}$. Celle-ci cherche à révéler l'« intersection » ou l'« entrecroisement » de divers facteurs générateurs de différence, qui mènent à des discriminations multidimensionnelles allant au-delà de l'addition de ses éléments. L'intersectionnalité postule l'interaction des catégories de sexe/genre, classe, race, ethnicité, âge, handicap et orientation sexuelle dans la production et la reproduction des inégalités sociales et propose d'appréhender "la réalité sociale des femmes et des hommes, ainsi que les dynamiques sociales, culturelles, économiques et politiques qui s'y rattachent comme étant multiples et déterminées simultanément et de façon interactive par plusieurs axes d'organisation sociale significatifs ${ }^{4}$ ».

Le premier mouvement féministe allemand s'attaquait aux discriminations liées à l'appartenance de sexe et se fondait pour cela, en particulier dans la phase de gestation, sur l'appartenance des femmes à l'espèce humaine. "Les droits humains n'ont pas de sexe »: telle était la formule trouvée par l'écrivaine et essayiste juive Hedwig Dohm (1831-1919). Cette lutte pour l'universalisme n'a pas empêché les femmes de développer par ailleurs la conception d'une « geistige/ organisierte Mütterlichkeit » (maternité sociale ou organisée), qui s'appuyait sur une différence supposée essentielle entre les sexes, et qui attribuait aux femmes un rôle particulier dans la communauté nationale. L'éducation, la transmission du savoir et le domaine des soins (ce qu'on appelle aujourd'hui le « care ») : tels furent les domaines d'expertise que les femmes s'attribuèrent au nom de qualités maternelles qu'hommes et femmes s'accordaient en général à leur prêter ${ }^{5}$. Les femmes engagées dans des associations travaillant dans ces domaines voyaient dans leurs activités un vecteur d'émancipation de l'idéal féminin de la société bourgeoise.

Il s'agit dans ce qui suit d'examiner dans quelle mesure les femmes juives se sont identifiées à cette conception de l'émancipation et ont participé au mouvement féministe allemand, et quel traitement celui-ci a réservé à l'appartenance religieuse de ses membres. Dans une Prusse-Allemagne à forte domination luthérienne, dans laquelle l'antisémitisme traditionnel, fondé sur des motifs religieux et culturels, a évolué au cours du XIX siècle en « code culturel » (Shulamit Volkov ${ }^{6}$ )

3. Matthias Bähr, Florian Kühnel, «Plaidoyer für eine historische Intersektionsanalyse », Zeitschrift für Historische Forschung 56, 2018, p. 9-37.

4. Daiva K. Stasiulis, "Feminist Intersectional Theorizing », in Peter S. Li (dir.), Race and Ethnic Relations in Canada, Toronto, Oxford UP, 1999, p. 347, cité par Sirma Bilge, "Théorisations féministes de l'intersectionnalité ", Diogène 225 (1), 2009, p. 70-88, DOI : 10.3917/dio.225.0070. En ligne : [https://www.cairn.info/revue-diogene-2009-1-page-70.htm], dernière consultation le 8 février 2019.

5. Heidemarie Wawrzyn, «Als die "Mütter der Nation“ Abschied nahmen von den Menschenrechten: Über Antisemitismus in den bürgerlichen Frauenbewegungen 1865-1918 », in Ute Planert (dir.), Nation, Politik und Geschlecht. Frauenbewegungen und Nationalismus in der Moderne, Frankfurt a. M., Campus, 2000, p. 182-197, ici p. 182-183.

6. Shulamit Volkov, «Antisemitismus als kultureller Code”, in id., Antisemitismus als kultureller Code:

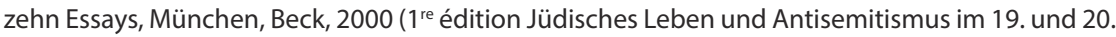
Jahrhundert, 1990), p. 13-36. 
ou « norme sociétale largement répandue » (Hermann Greive $\left.{ }^{7}\right)$, l'appartenance religieuse était pour le moins génératrice de différences. Le mouvement féministe allemand, qui lui-même luttait contre des mécanismes d'exclusion liés à une appartenance, a-t-il su résister à l'antisémitisme ambiant au nom de la solidarité féminine ou de l'universalisme dont il se réclamait?

Pour répondre à ces questions, nous commencerons par prendre la mesure de l'engagement de femmes juives dans le mouvement féministe allemand. La deuxième partie sera dédiée au Jüdischer Frauenbund, I'Union des associations de femmes juives en Allemagne créée en 1904 : nous examinerons ses objectifs et son évolution au sein du mouvement féministe allemand. Enfin, nous nous pencherons sur l'attitude des leaders du mouvement féministe allemand face à ses membres juifs et aux mécanismes d'exclusion et aux comportements antisémites dans la société comme dans leurs propres rangs.

\section{Forte participation des femmes juives dans le mouvement féministe allemand}

\section{Surreprésentation quantitative et qualitative}

Selon Irmgard Maya Fassmann, auteure d'une monographie sur les Juives dans le mouvement féministe allemand de 1865 à 1919, un tiers des principales actrices du mouvement féministe était d'origine juive, soit 20 parmi un groupe de 60 femmes identifiées comme actrices principales, les membres du Jüdischer Frauenbund étant mises à part ${ }^{8}$. Compte tenu du fait que les Juifs et Juives constituaient $1 \%$ de la population de l'Empire allemand ${ }^{9}$, les femmes juives étaient donc très nettement surreprésentées dans le mouvement féministe ${ }^{10}$. Notons par ailleurs que les places fortes du mouvement féministe allemand (Berlin, Hambourg, Leipzig et Francfort-sur-le-Main) étaient également des hautslieux de la communauté juive assimilée, avec par exemple 4,8\% de Juifs dans la population berlinoise et $7,6 \%$ dans celle de Francfort, selon le recensement de $1900^{11}$.

Sur le plan qualitatif également, les femmes juives jouaient un rôle important dans le mouvement féministe allemand : elles étaient nombreuses à la tête d'associations, au niveau local, régional et suprarégional. Irmgard Maya Fassmann cite plusieurs féministes juives occupant des postes de direction au sein du Bund Deutscher Frauenvereine (BDF, Union des associations féminines allemandes) :

7. « weitverbreitete gesellschaftliche Norm ». Hermann Greive, Geschichte des modernen Antisemitismus in Deutschland, Darmstadt, Wissenschaftliche Buchgesellschaft, 1983, p. 73.

8. Irmgard Maya Fassmann, Jüdinnen in der deutschen Frauenbewegung 1865-1919, Hildesheim, Georg Olms, 1996, p. $13 ; 16$.

9. Arno Herzig, Jüdische Geschichte in Deutschland von den Anfängen bis zur Gegenwart, Bonn, Bundeszentrale für politische Bildung, 2005, p. 196.

10. Fassmann, Jüdinnen, p. 293.

11. Ibid., p. 13. 
Alice Salomon (1872-1948) et Helene Simon (1862-1947) à la direction de la Commission pour la protection des ouvrières, Josephine Levy-Rathenau (1877-1921) à la tête de la Commission pour l'emploi, Alice Bensheimer (1864-1935) au poste de secrétaire du bureau, Jeanette Schwerin (1852-1899) et Anna Pappritz (1861-1939, bien qu'en réalité elle ne soit pas juive ${ }^{12}$ ) en charge de la rédaction de l'organe de presse du DBF, et Elisabeth Altmann-Gottheiner (18741930) comme éditrice du Jahrbuch der Frauenbewegung ${ }^{13}$. Cela laisse penser que les femmes juives étaient bien acceptées au sein du BDF.

Avec des personnalités telles que Fanny Lewald, Jenny Hirsch, Johanna Goldschmidt, Henriette Goldschmidt, Auguste Auerbach (épouse Steinschneider) - une génération de femmes nées entre 1810 et $1840^{14}$-, les femmes juives se sont engagées dès ses origines en grand nombre et avec une certaine évidence dans le mouvement féministe allemand. Comment expliquer qu'elles aient si largement adhéré au concept d'émancipation porté par le mouvement féministe?

\section{Motivations des femmes juives engagées dans le mouvement féministe}

Dans la tradition juive, l'organisation de la vie pratique au quotidien était du ressort des femmes, tandis que la tâche principale des hommes était l'étude de la Torah et du Talmud. La masculinité n'y était donc pas prioritairement définie par le travail physique et le rôle de pourvoyeur financier principal de la famille. Ce rôle pouvait même être endossé si nécessaire par l'épouse, sans que cela ne mette en question la hiérarchie entre les sexes et le patriarcat ${ }^{15}$.

Les jeunes filles de familles juives issues d'un milieu libéral urbain recevaient souvent une éducation très poussée (dans les premières écoles de filles juives ou étatiques et à domicile) ; elles souffraient ensuite à la fois de leur soif de savoir et de l'oisiveté démonstrative imposée aux femmes dans les milieux bourgeois. Les écrivaines féministes Hedwig Dohm (1831-1919) et Fanny Lewald (1811-1889) ont formulé ce problème dans leur œuvre littéraire ; quelques décennies plus tard, Henriette Fürth (1861-1938), Bertha Pappenheim (1859-1936), Alice Salomon, décrivaient dans leurs mémoires la même redoutable oisiveté de leurs années

12. Anna Pappritz n'était pas juive. Elle a reçu une éducation chrétienne et a vraisemblablement été baptisée dans l'Église protestante. Kerstin Wolff, Anna Pappritz 1861-1939. Die Rittergutstochter und die Prostitution, Sulzbach/Ts., Ulrike Helmer, 2017, p. 14 ; 22.

13. Fassmann, Jüdinnen, p. 264.

14. Helga Krohn, «Aufbruch in eine neue Gesellschaft? Erwartungen jüdischer Frauen an die deutsche Frauenbewegung und die Grenzen der Zusammenarbeit », in Mechthild Bereswill, Leonie Wagner (dir.), Bürgerliche Frauenbewegung und Antisemitismus, Tübingen, Diskord, 1998, p. 13-43, ici p. 19-20.

15. Monika Richarz, "Geschlechterhierarchie und Frauenarbeit seit der Vormoderne ", in Kirsten Heinsohn, Stefanie Schüler-Springorum (dir.), Deutsch-jüdische Geschichte als Geschlechtergeschichte. Studien zum 19. und 20. Jahrhundert, Göttingen, Wallstein, 2006, p. 87104, ici p. 88-89. 
de jeunesse après la sortie de l'école dans l'attente du mariage ${ }^{16}$. Cette soif de savoir et le besoin de se soustraire à l'impératif d'oisiveté constituaient une des motivations majeures pour les femmes issues de familles aisées, pour s'engager dans le mouvement féministe. Elles pouvaient ainsi se rendre utiles à la société et donner un sens à leur existence.

\section{Domaines de prédilection}

Les femmes juives trouvèrent dans le mouvement féministe deux domaines de prédilection : le mouvement Fröbel, mouvement pour une nouvelle pédagogie de la petite enfance, et le travail social.

Le mouvement pédagogique réformateur initié par Friedrich Fröbel (1782-1852), à l'origine des jardins d'enfants (Kindergärten) et de la pédagogie moderne développés au milieu du XIX siècle comme alternative aux garderies d'enfants (Kinderbewahranstalten), a exercé une immense force d'attraction sur les femmes juives. Pour ne citer que quelques personnalités saillantes parmi celles-ci, Johanna Goldschmidt (1806-1884) à Hambourg, Henriette Goldschmidt (1825-1920, non parente de la précédente) à Leipzig et Auguste Steinschneider (1823-1989) ou encore Lina Morgenstern (1830-1909) à Berlin, se sont engagées durablement dans ce mouvement et en ont proposé des prolongements ${ }^{17}$.

Le mouvement de Fröbel permettait aux femmes juives de s'épanouir tout en professionnalisant leurs pratiques dans un domaine, celui de l'éducation des enfants, qui leur revenait selon la tradition juive. L'éducation morale et intellectuelle des enfants est même devenue, à côté de la tenue du foyer, la tâche spécifique des femmes dans le processus d'acculturation des juifs, enclenché dans la seconde moitié du XVIII ${ }^{e}$ siècle ${ }^{18}$. Le concept de Friedrich Fröbel, pour qui l'amour maternel prédestinait les femmes à une tâche aussi importante que l'éducation des enfants, faisait donc écho au rôle traditionnel des femmes juives et leur permettait par ailleurs de transposer à la société leurs obligations envers la communauté. Mais surtout, ce qui le rendait particulièrement attrayant aux yeux des femmes juives était que Fröbel proposait une éducation commune aux enfants de diverses origines sociales et religieuses, aspirant à dépasser les barrières sociales à l'égard des Juifs et à former une société à visage humain ${ }^{19}$.

Les femmes juives actives dans le mouvement Fröbel aspiraient donc à contribuer à relativiser les différences religieuses, à abolir les barrières sociales et religieuses, et à œuvrer au progrès social. Or, dans la pratique, le dépassement des barrières sociales et religieuses par la pédagogie fröbelienne et l'intégration sociale à travers la coopération de femmes juives et chrétiennes se sont avérés bien difficiles ${ }^{20}$. Néanmoins, nombreuses sont les femmes juives qui sont entrées

16. Krohn, « Aufbruch in eine neue Gesellschaft? », p. 22-23.

17. Fassmann, Jüdinnen, p. 129-222.

18. Richarz, Geschlechterhierarchie, p. 94-95.

19. Fassmann, Jüdinnen, p. 132.

20. Krohn, «Aufbruch in eine neue Gesellschaft? », p. 31. 
en contact avec le mouvement féministe par le biais de leur engagement dans le mouvement Fröbel ${ }^{21}$.

La charité organisée et, dans sa version plus moderne, le travail social (soziale Arbeit) comme profession ont également exercé une forte attraction sur les femmes juives. La charité occupe une place importante dans le judaïsme, qui commande aux plus aisés d'apporter assistance aux pauvres, ceux-ci n'étant pas considérés comme des assistés, mais comme des êtres humains à qui il convient de rendre justice (Zedaka) et de montrer le chemin de l'autonomie ${ }^{22}$. Les activités caritatives allaient de visites de malades, de veufs et veuves à l'assistance hospitalière, et étaient pour les jeunes filles les seules activités autorisées en dehors du foyer familial. Ainsi, elles permettaient aux jeunes femmes de quitter momentanément l'espace familial, tout en exerçant une activité d'un grand prestige social. Les associations caritatives féminines étaient donc parfaitement en accord avec l'éthique sociale juive et sa conception de la charité comme un accompagnement vers l'autonomie (Hilfe zur Selbsthilfe), un concept également central dans le mouvement féministe allemand.

Une autre raison expliquant l'engouement des femmes juives pour le travail social est le fait que celles-ci, en raison de l'antisémitisme présent dans les directions d'établissements scolaires et dans le corps enseignant, n'avaient de fait que très peu de chances d'obtenir un poste d'institutrice, un métier souvent convoité par les femmes.

Tout ceci explique la participation massive des femmes juives ou d'ascendance juive dans les associations caritatives, avec une forte disposition au don et une volonté de moderniser et rationaliser les œuvres de charité pour évoluer vers le travail social ${ }^{23}$. Jeanette Schwerin et son élève Alice Salomon, à qui revient la maternité de la professionnalisation du travail social, sont deux figures pionnières dans ce domaine.

Fortes de leur activité associative, de nombreuses femmes conçurent une identité juive séculaire, mais l'élan émancipateur ne se limitait pas aux femmes d'orientation religieuse libérale. Très attachée aux traditions juives et y voyant les ressources nécessaires pour l'émancipation des femmes, Bertha Pappenheim allait opérer un changement de paradigme dans la participation des femmes juives au mouvement féministe allemand.

21. Fassmann, Jüdinnen, p. 130.

22. Krohn, « Aufbruch in eine neue Gesellschaft? », p. 32.

23. Ibid., p. 32-33. 


\section{Le Jüdischer Frauenbund ou le numéro d'équilibriste entre identité et différence}

\section{Contexte d'émergence du Jüdischer Frauenbund}

L'Union des associations féminines juives (Jüdischer Frauenbund, JFB) a été créée notamment pour lutter contre la traite des filles. La prostitution des jeunes filles et des femmes juives avait en effet fortement augmenté depuis le tournant du siècle, avec l'arrivée en grand nombre de Juif-ve.s d'Europe de l'Est ayant émigré en Allemagne ou s'y trouvant de passage. Nombreuses étaient les juives célibataires qui se prostituaient par nécessité économique, souvent après avoir été leurrées par des trafiquants déguisés en policiers ou en rabbins leur promettant de trouver pour elles un lieu de séjour, un emploi ou un époux ${ }^{24}$. Afin de ne pas apporter de l'eau au moulin de la presse antisémite, les organisations juives taisaient ce sujet, et il fallut attendre 1902 pour que Bertha Pappenheim, bénévole expérimentée dans l'assistance communale aux pauvres et aux orphelins et fondatrice avec Henriette Fürth de la « Weibliche Fürsorge » (Assistance féminine), mette le sujet à l'agenda d'une rencontre de rabbins à Francfort-sur-le-Main ${ }^{25}$.

Le Jüdischer Frauenbund doit également sa création à l'évolution du secteur des œuvres de charité (Wohlfahrt) en travail social (soziale Arbeit). Le besoin se faisait ressentir de moderniser les œuvres de charité juives. De jeunes femmes juives critiquaient les bonnes œuvres de leurs mères, des initiatives privées se caractérisant par un certain dilettantisme en ce qu'elles apportaient de l'assistance sans traiter le problème à la racine. Cette jeune génération de femmes se forma dans écoles dédiées, comme la Soziale Frauenschule créée à Berlin par Alice Salomon, avec le soutien d'universitaires et d'experts. Cette volonté de professionnalisation allait de pair avec la sécularisation du mode de vie des femmes juives issues de familles aisées d'orientation libérale. Ces femmes étaient moins liées à leur communauté et davantage tournées vers la commune/ la ville (Gemeinde), où le travail social justement se dotait de structures et se professionnalisait.

Les femmes juives engagées dans le travail social aspiraient à une plus grande participation dans la société. Dans leur élan émancipateur, elles aspiraient également à davantage de capacité d'action au sein de la communauté religieuse, où elles étaient privées du droit de vote. Bertha Pappenheim entendait également, et cela était nouveau, allier la participation au mouvement féministe et l'affirmation en son sein de l'appartenance et de l'identité juives.

24. Gudrun Maierhof, « Jüdischer Frauenbund », in Dan Diner (Hg.), Enzyklopädie jüdischer Geschichte und Kultur, vol. 3, Stuttgart/ Weimar, Metzler, 2012, p. 255-259, ici p. 256.

25. Ibid., Pour plus de détails Britta Konz, Bertha Pappenheim (1859-1936). Ein Leben für jüdische Tradition und weibliche Emanzipation, Frankfurt a. M./ New York, Campus, 2005, p. 81-85. 


\section{Les objectifs du Jüdischer Frauenbund}

Depuis la création d'une Union des associations féminines évangéliques (Deutsch-evangelischer Frauenbund, DEF) en 1899 et de son pendant catholique (Katholischer Frauenbund, KFB) en 1903, les femmes protestantes et catholiques étaient représentées par des associations qui faisaient valoir leur appartenance religieuse. Les associations de femmes juives s'inspirèrent de ces modèles et firent de même un an plus tard. Le Jüdischer Frauenbund (JFB) vit le jour le 15 juin 1904 à Berlin, à l'occasion du congrès du Conseil international des femmes qui s'y tenait. Aux côtés de Bertha Pappenheim, co-fondatrice et présidente du JFB jusqu'en 1934, et de Sidonie Werner, sa vice-présidente, Henriette May (18621928), Paula Ollendorff (1860-1938), Ottilie Schönewald (1883-1961), Hannah Karminski (1897-1943) et Cora Berliner (1890-1942) comptaient parmi les actrices les plus importantes de cette organisation ${ }^{26}$.

Dans ses statuts, le Jüdischer Frauenbund définit son activité de la façon suivante :

a) In gegenseitiger Anregung für die Interessen der Allgemeinheit. Als Arbeitsgebiete gelten zunächst Wege und Ziele sozialer Hilfstätigkeit, der Volkserziehung, der Förderung des Erwerbslebens jüdischer Frauen und Mädchen, Hebung der Sittlichkeit, Bekämpfung des Mädchenhandels; b) im Erwecken des Interesses zu allgemeinen jüdischen Bestrebungen der Gegenwart und durch Stärkung des jüdischen Gemeinschaftsbewußtseins ${ }^{27}$.

Il s'agissait donc, premièrement, de lutter contre la prostitution et la traite des filles, en priorité de celles immigrées d'Europe de l'Est. Le JFB a déployé un réseau de bureaux d'accueil dans les gares et les ports pour les jeunes filles voyageant seules, érigé des foyers pour les mères célibataires victimes de la traite des filles, afin d'éloigner mère et enfants de ces réseaux, mené des campagnes d'information à l'adresse des jeunes femmes en Allemagne pour prévenir l'entrée dans la prostitution.

Le JFB aspirait également à promouvoir l'activité professionnelle des jeunes filles et femmes juives, notamment pour prévenir la prostitution pour raisons économiques. Il a développé pour cela une offre de formation considérable dans le domaine du travail social et des tâches domestiques, mis en place des bureaux d'offres d'emploi, et promu l'activité féminine comme un moyen de parvenir à l'indépendance économique, psychologique et émotionnelle.

Le JFB luttait aussi pour que les femmes puissent prendre part aux décisions dans la communauté religieuse. II réclamait aussi bien le droit de vote des femmes dans la communauté que dans la cité, contrairement aux fédérations protestantes et catholiques : la première étant très réservée quant aux droits

26. Marion A. Kaplan, Die jüdische Frauenbewegung in Deutschland. Organisation und Ziele des Jüdischen Frauenbundes 1904-1938 (titre original : The Jewish Feminist Movement in Germany: the Campaigns of the Jüdische Frauenbund, 1904-1938, 1979), Hamburg, Hans Christians Verlag, 1981, p. 141-153.

27. Cité dans Christina Klausmann, Politik und Kultur der Frauenbewegung im Kaiserreich. Das Beispiel Frankfurt am Main, Frankfurt a. M./ New York, Campus, 1997, p. 120. 
civiques et la seconde ne s'étant jamais prononcée sur la question ${ }^{28}$. Ce combat pour la participation politique au sein de la communauté était, selon Marion Kaplan, le combat le plus difficile du JFB. Une fois le droit de vote accordé aux femmes en Allemagne en 1918, les femmes du JFB insistèrent pour obtenir également le droit de participer aux décisions prises dans la communauté, mais il leur fallut attendre jusqu'à la fin des années 1920 pour l'obtenir, du moins dans six des sept plus grandes villes allemandes. Elles n'avaient certes toujours pas gagné en influence ni en pouvoir, mais elles détenaient l'égalité formelle ${ }^{29}$.

Toutes ces luttes et initiatives visaient enfin à consolider le sentiment d'appartenance à la communauté juive et à renforcer la confiance en elle des femmes juives, qui s'émancipaient et se réalisaient à travers leurs activités dans la sphère publique ${ }^{30}$. Il s'agissait donc pour elles d'affirmer leur appartenance religieuse de manière positive. Quant à se faire accepter en tant que femmes juives ou d'ascendance juive dans la société bourgeoise, elles n'étaient pas maîtresses de la situation.

\section{Rapports du mouvement féministe allemand avec ses membres juifs}

\section{Stratégies d'invisibilisation et d'exclusion}

Le Jüdischer Frauenbund compta bientôt un grand nombre d'adhérentes : y étaient membres environ $20 \%$ de toutes les femmes juives âgées de plus de 21 $a^{31}{ }^{31}$. Le JFB s'affilia au Bund deutscher Frauenvereine (BDF) dès 1907, soit un an avant I'Union évangélique (DEF) ; I'Union catholique (KFB), quant à elle, ne s'y affilia jamais. Par son affiliation, le JFB enrichissait le BDF de 120 associations. Bertha Pappenheim, sa présidente, siégea également au bureau du BDF de 1914 à 1924 ; d'autres féministes juives comme Henriette Fürth étaient également engagées dans les deux fédérations, BDF et JFB ${ }^{32}$. Leur volonté d'intégration était donc manifeste. Le JFB poursuivait - intérêts spécifiques mis à part - les mêmes objectifs que le BDF et il attendait de lui un soutien dans la lutte contre les préjugés et propos antisémites. Or, le BDF affichait certes une neutralité confessionnelle dans ses statuts, mais il se considérait naturellement comme chrétien. On peut donc se demander comment les féministes ont perçu l'appartenance à la religion juive de certains de leurs principaux membres, et comment elles ont réagi face aux comportements antisémites venant de l'extérieur ou de leurs propres rangs.

28. Heidemarie Wawrzyn, Vaterland statt Menschenrecht: Formen der Judenfeindschaft in den Frauenbewegungen des Deutschen Kaiserreiches, Marburg, diagonal-Verlag, 1999, p. 160.

29. Marion Kaplan, "Schwesterlichkeit auf dem Prüfstand. Feminismus und Antisemitismus in Deutschland, 1904-1938 », Feministische Studien 3, 1984, p. 128-149, ici p. 131.

30. Maierhof, Jüdischer Frauenbund, p. 256.

31. Krohn, « Aufbruch in eine neue Gesellschaft? », p. 37.

32. Wawrzyn, Vaterland, p. 214. 
Analysant le positionnement du BDF face aux comportements antisémites émanant du mouvement féministe allemand, Heidemarie Wawrzyn a montré que :

Die "Schwesterlichkeit" wurde in Krisenzeiten von den nichtjüdischen Mitgliedern der Frauenbewegung schnell aufgekündigt. Die bevorzugten "Strategien" des Bundes Deutscher Frauenvereine, sich jeder Parteinahme für jüdische Mitglieder zu entziehen, waren Bagatellisierung und Formalisierung, Schweigen bzw. Nichterwähnung sowie taktisches, informelles Intrigieren. An der "groben" und "unkultivierten" Art antisemitischer Äußerungen wurde Anstoß genommen, jedoch nicht an antijüdischen Einstellungen und Verhaltensweisen. Die Mehrheit des Bundes hatte offensichtlich den Antisemitismus im Kaiserreich akzeptiert ${ }^{33}$.

Présidente du BDF de 1910 à 1919, Gertrud Bäumer (1873-1954) peinait à reconnaître le Jüdischer Frauenbund comme faisant partie du mouvement féministe allemand; dans ses discours, elle ne mentionnait pas son nom ${ }^{34}$. Helene Lange (1848-1930), présidente de I'ADF depuis 1902, lors de son allocution à l'occasion du $50^{\mathrm{e}}$ anniversaire de l'ADF en 1915, passa également sous silence l'existence du JFB lorsqu'elle évoqua la coopération de longue date entre le BDF et les associations féminines confessionnelles ${ }^{35}$. Ce faisant, Bäumer et Lange privaient le JFB de la visibilité et de la reconnaissance auxquelles précisément il aspirait.

Quant aux stratégies de minimisation et de formalisation mentionnées par Heidemarie Wawrzyn, un exemple suffira à les illustrer : le $1^{\text {er }}$ mars 1914 parut dans l'Evangelische Frauenzeitung, l'organe de presse du DEF, le compte rendu d'une brochure intitulée Der deutsche Verfall (Le déclin allemand) signée d'Adolf Bartels (1862-1945), écrivain et journaliste völkisch, antisémite et antidémocrate. Rendant compte du point de vue de Bartels selon lequel pour prévenir le « déclin moral » du peuple allemand, il fallait vaincre le « libéralisme enjuivé », l'auteure n'exprima aucune réserve sur ces propos ${ }^{36}$. Alice Bensheimer, secrétaire au bureau du BDF, fit part de sa perplexité à Paula Müller, présidente du DEF, et à Gertrud Bäumer, présidente du BDF. Dans sa réponse, cette dernière excusa Paula Müller et tenta d'apaiser Alice Bensheimer en supposant que la présidente du DEF n'avait certainement pas lu le compte rendu (des fautes de frappe pouvaient le laisser penser). Bäumer tenta de balayer l'incident d'un revers de la main en remarquant que les propos de Bartels n'étaient qu'une série d'inepties. Pour finir, elle disait regretter devoir respecter, en qualité de présidente du BDF, le principe de noningérence dans les affaires des associations affiliées : le recours à des formalités lui permit de se soustraire à l'obligation de protester. Enfin, dans une lettre adressée cette fois à la comtesse Selma von der Groeben, vice-présidente du DEF, Bäumer précisa que ce qui la dérangeait, dans l'expression « libéralisme enjuivé », était non pas son contenu antisémite, mais l'attaque du libéralisme, sa famille

33. Ibid., p. 219.

34. À ce sujet, voir Marlis Dürkop, «Erscheinungsformen des Antisemitismus im Bund deutscher Frauenvereine », Feministische Studien 3, 1984, p. 140-164; Wawrzyn, Vaterland, p. 203-219.

35. Wawrzyn, Vaterland, p. 60.

36. Evangelische Frauenzeitung 14, 1914, p. 86. 
politique ${ }^{37}$. Un an plus tôt, Bäumer avait recouru aux mêmes stratégies lorsque Henriette Goldschmidt avait subi à Leipzig une diffamation à caractère antisémite dans la presse ${ }^{38}$. Tout cela tend à montrer qu'une personnalité aussi influente sur le mouvement féministe que Gertrud Bäumer avait non seulement accepté l'antisémitisme, mais participait aussi aux mécanismes d'exclusion antisémites.

\section{Céder à l'antisémitisme pour des raisons stratégiques}

De manière générale, le BDF, y compris son aile "radicale ", plus sensible aux questions sociales, mais également plus franchement laïque, se gardait de réagir aux manifestations d'antisémitisme venues de l'extérieur ${ }^{39}$. Sans être de farouches antisémites, les femmes à la tête du BDF cédèrent parfois à l'antisémitisme pour des raisons stratégiques.

L'élection de la nouvelle présidente du BDF en 1914/1919 en dit long à ce sujet: lorsque, en 1913-1914, Alice Salomon fut fortement pressentie pour accéder à la présidence du BDF, la fédération évangélique (DEF) exprima des réserves quant à l'élection d'une "Israélite » à ce poste. Or Alice Salomon allait se convertir au protestantisme en août 1914, et les membres du bureau du DEF le savaient. En raison de la guerre, l'élection fut repoussée jusqu'en 1919. Ce n'est finalement pas Alice Salomon, mais Marianne Weber (1870-1954), qui fut élue. Pendant que les discussions internes se poursuivaient, Gertrud Bäumer avait approché celle-ci et avait fait en sorte qu'elle soit élue. Or, il est avéré que, sur le plan personnel, Bäumer aurait préféré qu'Alice Salomon prît la présidence du BDF, mais qu'elle a appuyé la candidature de Marianne Weber pour ne pas mettre en danger la cohésion interne du BDF et faire bonne figure vers l'extérieur ${ }^{40}$. Des considérations de pouvoir et d'image ont donc guidé ce choix. Pour les mêmes raisons, à partir de 1914, les femmes ayant " un nom ou des origines juives » n'étaient plus les bienvenues pour prendre la parole lors des assemblées du BDF ${ }^{41}$.

\section{Détérioration de la situation après 1914}

L'engagement commun de femmes juives et chrétiennes pour une société plus humaine fut lourdement grevé par la montée d'un nationalisme de plus en plus agressif et d'un antisémitisme fondé sur des pseudo-théories raciales. La première guerre mondiale ne fit qu'accentuer les phénomènes cités et renforcer le caractère paradoxal des relations entre femmes juives et non-juives au sein du

37. Wawrzyn, Vaterland, p. 214-215.

38. Ibid., p. $62 ; 217$.

39. Anne-Laure Briatte, «Wie radikal war die radikale Frauenbewegung im Umgang mit (antifeministischem) Antisemitismus? », in Frauen \& Geschichte Baden-Württemberg e.V., Liselotte Homering, Sybille Oßwald-Bargende, Mascha Riepl-Schmidt, Ute Scherb (dir.), AntisemitismusAntifeminismus, Ausgrenzungsstrategien im 19. und 20. Jahrhundert, Sulzbach/Ts., Helmer, 2019 (à paraître).

40. Wawrzyn, Vaterland, p. 218.

41. Ibid., p. 219. 
mouvement féministe. Tels les hommes juifs qui se sont engagés dans la guerre avec élan, y voyant une occasion de fournir la preuve de leur loyauté envers la nation allemande, les femmes juives ont contribué à l'effort féminin de guerre dans le Service national des femmes ${ }^{42}$. Dans le même temps, le recensement des juifs de 1916 (« Judenzählung ») rappelait aux Juifs et aux Juives leur exclusion de la communauté nationale. Au lendemain de la guerre, la concurrence pour les places dans les universités et les emplois nourrissait les ressentiments antisémites, alors que les dernières étapes de la lutte pour le suffrage des femmes avaient eu sur elles un effet fédérateur ${ }^{43}$.

En 1920, le JFB redéfinit et précisa ses objectifs : "L'association veut réaliser son objectif : a) en renforçant le sentiment d'appartenance à la communauté » et, après une longue liste d'objectifs sociaux, pacifistes et féministes, il terminait avec « k) en luttant contre toutes les formes d'antisémitisme ${ }^{44}$ ». En dépit des signes indiquant que le BDF, à partir de 1918-1919 adoptait ici et là des comportements antisémites, le JFB attendait de lui un soutien dans la lutte contre l'antisémitisme. À partir de 1930, le BDF soutint effectivement les campagnes d'information menées par le JFB visant à faire reculer le fanatisme. Agnes von Zahn-Harnack (1884-1950), présidente du BDF depuis 1931, reconnaissant que « la lutte contre l'antisémitisme [devait] venir des femmes chrétiennes ${ }^{45}$ ", émit des propositions pour un rapprochement entre femmes chrétiennes et juives. Mais ces propositions aussi bien que les soirées d'information et les brochures diffusées, les visites de synagogues organisées par le JFB, et les pamphlets contre les nazis rédigés par le BDF (Gelbe Blätter ${ }^{46}$ ) semblent, avec le recul, bien peu adaptés à la virulence des ressentiments contre les Juifs et Juives.

Ces moyens ne suffirent pas à endiguer l'antisémitisme, loin s'en faut. Après I'accession des nazis au pouvoir en 1933, le BDF fut sommé par Lydia Gottschewski (1906-1989), présidente de la NS-Frauenschaft, de démettre toutes les femmes juives de leurs fonctions au bureau et d'élire à leur place des adeptes du nazisme ; de plus, le BDF devait s'affilier au Front allemand des Femmes (Deutsche Frauenfront ${ }^{47}$ ). Les statuts du BDF ne permettant pas son affiliation à une autre organisation, le bureau décida la dissolution du BDF le 15 mai 1933. De 1933 à 1938 , le JFB voua son activité à la survie de la communauté juive, notamment en organisant l'émigration des femmes à partir de 1935. Dans le sillon du pogrom de novembre 1938, le JFB fut interdit ; certaines de ses activistes majeures, comme Hannah Karminski et Cora Berliner, qui n'avaient pas quitté le pays et s'étaient

42. Kaplan, Schwesterlichkeit, p. 132.

43. Krohn, " Aufbruch in eine neue Gesellschaft? », p. 40-41.

44. «Der Verein will seinen Zweck erreichen: a) durch Stärkung des Gemeinschaftsbewußtseins (...) k) durch Bekämpfung aller Formen des Antisemitismus. » Kaplan, Die jüdische Frauenbewegung, p. 123.

45. «Der Kampf gegen Antisemitismus muß von den christlichen Frauen ausgehen. » Cit. dans Kaplan, Schwesterlichkeit, p. 133.

46. Ibid.

47. Wawrzyn, Vaterland, p. 210-211. 
encore efforcées de venir en aide aux juives en Allemagne, furent déportées et assassinées ${ }^{48}$.

Partagées entre leur sentiment d'identité et de différence, les féministes juives se sont très largement identifiées aux objectifs du mouvement féministe allemand et l'ont attesté à travers un engagement fort et durable. Selon Marion Kaplan, elles étaient néanmoins confrontées à un "dilemme propre aux minorités modernes : le souhait d'être accepté par la majorité et de conserver en même temps sa singularité propre ${ }^{49}$ ». Avec la création du Jüdischer Frauenbund, il s'agissait, au-delà de cette identité de vues et de sentiments, d'affirmer également leur différence, cette autre appartenance qui était la leur, et d'accéder à la reconnaissance dans la société. La double affiliation de mainte féministe juive, par exemple au bureau du JFB et du BDF, reflétait leur double appartenance à des identités qui se recouvraient largement, tout comme les objectifs du JFB et du BDF se recouvraient en grande partie. Or les féministes juives firent l'expérience de l'indifférence parfois hostile des féministes chrétiennes pour leurs intérêts et leurs luttes propres. Plus exactement, les féministes juives étaient d'autant plus appréciées qu'elles ne mettaient pas en avant leur identité juive. ${ }^{50}$ Sous l'influence d'un nationalisme blessé et de fortes tensions sociales et économiques, le mouvement féministe allemand a, sauf exception, progressivement cédé du terrain à l'antisémitisme.

Au terme de son étude sur les femmes juives dans le mouvement féministe allemand de 1865 à 1919, Irmgard Fassmann conclut que les féministes juives et non-juives entretenaient des relations correctes, voire amicales, en période calme, mais que, à quelques exceptions près, leurs relations se détérioraient rapidement en temps de crise. Alors les féministes non-juives, craignant pour elles des retombées négatives, se gardaient bien de se solidariser avec les juives ${ }^{51}$. Le fait est que, depuis les années 1910, lorsque le mouvement féministe - et ouvrier - gagnait en ampleur, que la perspective d'une démocratisation du système politique se dessinait, le mouvement féministe dans son ensemble est devenu la cible d'attaques antiféministes, antilibérales et antisémites, indépendamment, parfois, de la religion des personnes visées. Vu sous cet angle, le mouvement féministe fut, à partir de ce moment, en état de crise permanente, et les femmes non-juives ont parfois estimé plus opportun de se distancer ostensiblement d'une catégorie (religion) d'autant plus génératrice de différences que les théories antisémites pseudo-raciales opéraient, dans leur effort de biologisation, un glissement de l'appartenance religieuse à l'appartenance ethnique (« race»). Les féministes juives étaient donc exposées à des discriminations multidimensionnelles spécifiques que ne connaissaient pas les féministes chrétiennes, et face auxquelles toutes étaient impuissantes.

48. Maierhof, Jüdischer Frauenbund, p. 259.

49. Kaplan, Schwesterlichkeit, p. 129.

50. Ibid., p. 134.

51. Fassmann, Jüdinnen, p. 296-297. 
